\title{
Does pIgR Down-Regulation in COPD Cause Reprogramming of Bronchial Epithelium?
}

\author{
Spoorthi Sankineni $\cdot$ Young Cho $\cdot$ Nima Hosseinian • \\ Narasaiah Kolliputi
}

Received: 26 September 2014 / Accepted: 9 November 2014/Published online: 20 November 2014

(c) Springer Science+Business Media New York 2014

Chronic obstructive pulmonary disease (COPD) is a wellknown respiratory disease characterized by obstruction of airflow and progressive airway/lung inflammation secondary to harmful stimuli. Although it is a preventable and treatable disease, there is no cure [1]. It is associated with considerable morbidity and is predicted to become the third most common cause of mortality [2]. Cigarette smoking is the main risk factor for COPD; it leads to airway inflammation and alters epithelium through neutrophilic inflammation, mucosal hyperplasia, subepithelial fibrosis, and squamous metaplasia [3, 4].

Patients with COPD have an increased risk for infections, mainly due to impaired mucociliary clearance and decreased phagocytic function of alveolar macrophages [5]. Secretory Immunoglobulin A (SIgA) is the mainstay protective mechanism against infections of mucosal surfaces. SIgA is composed of two monomeric IgA components, J polypeptide chain, and a secretory component (SC). The $\mathrm{SC}$ is from the extracellular domain of the polymeric immunoglobulin receptor (pIgR). PIgR allows for transcytosis across the epithelial cell. Recent research suggests that SIgA acts a defense against infection by preventing the adhesion of pathogens to mucosal surfaces, a process known as "immune exclusion" [5].

A previous study showed decreased $\mathrm{pIgR/SC}$ expression in epithelial cells of severe COPD patients due to neutrophilic infiltration [6]. However, there was an increase in expression of pIgR when bronchial epithelial cells were

\footnotetext{
S. Sankineni · Y. Cho · N. Hosseinian · N. Kolliputi $(\bowtie)$ Division of Allergy and Immunology, Department of Internal Medicine, Morsani College of Medicine, University of South Florida, 12901 Bruce B. Downs Blvd., MDC 19, Tampa, FL 33612, USA

e-mail: nkollipu@health.usf.edu
}

cultured with TGF- $\beta$ [7] or supernatants from activated neutrophils [6]. In addition to these studies, there is abundant research regarding the stimuli for pIgR expression and upregulation [8-10]. However, the mechanisms for the decreased expression of pIgR have not been elucidated until a recent study by Gohy et al.

The study addressed whether down-regulation of pIgR in COPD was associated with disease severity. The study consisted of 116 patients, including controls, both nonsmoker and smoker, and patients with each stage of COPD. Human broncho-epithelial cells (HBEC) were obtained from an area of bronchus distant to the tumor site for culture in air/liquid interface (ALI) to allow for re-differentiation into mucociliary airway epithelium [4]. These cultures were treated with cigarette smoke extract in the apical compartment and TGF- $\beta 1$ in the basolateral compartment. Immunohistochemistry staining for pIgR/SC, phospho-SMAD $2 / 3$, and TGF- $\beta 1$ was performed on serial paraffin lung sections. HBEC filter paraffin sections were stained for pIgR/SC and various epithelial and mesenchymal markers. Researchers analyzed the staining intensity, percent of stained area, and localization of the staining within the epithelium. Western blot and ELISA were used to evaluate the expression of pIgR/SC, SC, and TGF- $\beta 1$. Researchers isolated and reverse transcribed the total RNA from lung tissue to quantify the expression of pIgR/SC and TGF- $\beta 1$ through real-time quantitative PCR [4]. Epithelial IgA transcytosis capacity in both ALI and submerged cultures after incubation with dimeric IgA was also assessed [4].

The study by Gohy et al. suggests that there is decreased expression of $\mathrm{pIgR}$ in the bronchial epithelium of patients suffering from severe COPD. Compared to non-smokers, severe COPD patients showed decreased staining intensity and area of pIgR in the large airways. Decreased pIgR expression was only statistically significant in patients with 
Fig. 1 The diagram is a representation of the proposed mechanism for Polymeric Immunoglobulin Receptor (pIgR) in Chronic Obstructive Pulmonary Disease (COPD)

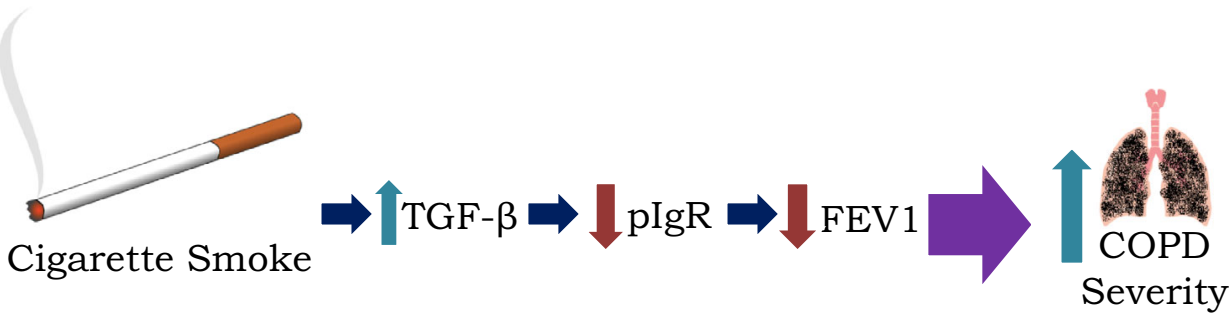

severe disease and not the mild disease. The decrease in pIgR expression correlated with a decrease in FEV1, and therefore was associated with airflow limitation. In smokers and patients with mild COPD, gene expression in proximal airways revealed an increase in transcription of $\mathrm{pIgR}$ when compared to non-smokers/controls. This relative upregulation was not seen in patients with moderate to severe COPD. These data suggest that decreased $\mathrm{pIgR}$ due to COPD indicates disease severity while smoking and COPD result in opposing effects on pIgR gene transcription [4].

To further assess the relation between COPD and $\mathrm{pIgR} / \mathrm{SC}$ production, the study compared the expression of $\mathrm{pIgR/SC}$ by in vitro bronchial epithelium of COPD patients to ex vivo. Similar to ex vivo findings, there was decreased pIgR immunostaining in moderate to severe COPD, pIgR down-regulation that correlated with limitations of airflow, and decreased transcytosis capacity of SIgA [4]. Therefore, it was concluded that with severe COPD, pIgR expression in bronchial epithelium reconstituted in vitro was downregulated (Fig. 1).

Researchers also evaluated the mechanism behind the down-regulation of pIgR and found an increased amount of TGF- $\beta 1$ released by ALI-HBEC in patients with severe COPD. When ALI-HBEC were incubated with TGF- $\beta 1$, there was a decrease in pIgR immunoreactivity and a "dedifferentiation" of the epithelium due to an induction of mesenchymal makers which lowered the levels of cytokeratins and E-cadherins. In these epithelial cells, there was also a decreased release of SC. This led to a decrease in pIgR mRNA and ultimately caused a decrease in the transcytosis capacity of IgA. Inhibiting TGF- $\beta 1$ in HBEC at the time of ALI differentiation led to a dose-dependent increase in $\mathrm{pIgR}$ expression [4].

The authors concluded that in severe COPD, downregulation of $\mathrm{pIgR}$ corresponds with airflow limitation and that increased TGF- $\beta 1$ expression can cause a decrease in pIgR and alter epithelial differentiation even when exposed in vitro [4]. They suggest the connection between TGF- $\beta$ signaling and $\mathrm{pIgR}$ expression should be further assessed in vitro in relation to various other pathways that are activated in COPD because the proposed mechanism provides insight that targeting $\mathrm{pIgR}$ may have a beneficial effect on patients with COPD and in turn may provide hope in developing therapeutic treatments to manage this severe and debilitating disease. Lastly, the experiment by Gohy et al. provides a framework for further research of the role of TGF- $\beta 1$ not only in COPD but also in other forms of lung disease, such as non-small cell lung cancer, that involve $\mathrm{pIgR}$ down-regulation.

Acknowledgements Narasaiah Kolliputi was funded by the American Heart Association National Scientist Development Grant 09SDG2260957 and National Institutes of Health R01 HL105932 and the Joy McCann Culverhouse Endowment to the Division of Allergy and Immunology.

Conflict of interest There is no conflict of interest.

\section{References}

1. Slok AH, In 't Veen JC, Chavannes NH et al (2014) Effectiveness of the Assessment of Burden of Chronic Obstructive Pulmonary Disease $(\mathrm{ABC})$ tool: study protocol of a cluster randomised trial in primary and secondary care. BMC Pulm Med 14:131

2. Stallberg B, Janson C, Johansson G et al (2014) Management, morbidity and mortality of COPD during an 11-year period: an observational retrospective epidemiological register study in Sweden (PATHOS). Prim Care Respir J 23:38-45

3. Davidsen PK, Herbert JM, Antczak P et al (2014) A systems biology approach reveals a link between systemic cytokines and skeletal muscle energy metabolism in a rodent smoking model and human COPD. Genome Med 6:59

4. Gohy ST, Detry BR, Lecocq M et al (2014) Polymeric Immunoglobulin Receptor Down-regulation in Chronic Obstructive Pulmonary Disease. Persistence in the Cultured Epithelium and Role of Transforming Growth Factor-beta. Am J Respir Crit Care Med 190:509-521

5. Bengoechea JA (2011) Secretory IgA and COPD: a new kid on the block? Am J Respir Crit Care Med 184:285-287

6. Pilette C, Godding V, Kiss R et al (2001) Reduced epithelial expression of secretory component in small airways correlates with airflow obstruction in chronic obstructive pulmonary disease. Am J Respir Crit Care Med 163:185-194

7. Ratajczak C, Guisset A, Detry B et al (2010) Dual effect of neutrophils on $\mathrm{pIgR} / \mathrm{sec}$ etory component in human bronchial epithelial cells: role of TGF-beta. J Biomed Biotechnol 2010

8. Pal K, Kaetzel CS, Brundage K et al (2005) Regulation of polymeric immunoglobulin receptor expression by reovirus. J Gen Virol 86:2347-2357

9. Ai J, Tang Q, Wu Y et al (2011) The role of polymeric immunoglobulin receptor in inflammation-induced tumor metastasis of human hepatocellular carcinoma. J Nat Cancer Inst 103:1696-1712

10. Rincheval-Arnold A, Belair L, Cencic A et al (2002) Up-regulation of polymeric immunoglobulin receptor mRNA in mammary epithelial cells by IFN-gamma. Mol Cell Endocrinol 194:95-105 